\title{
Intelligent Decision-Making System for Martial Arts Competition Using Deep Learning
}

\author{
Shisen Li, ${ }^{1}$ Shenglu Huo $\mathbb{D}^{2}{ }^{2}$ and Wei $\mathrm{Ke}^{3}$ \\ ${ }^{1}$ Department of Physical Education, North China University of Science and Technology, Tangshan 063210, Hebei, China \\ ${ }^{2}$ School of Economic and Management, Shanghai University of Sport, Shanghai 200438, China \\ ${ }^{3}$ College of Physical Education, HengShui University, Hengshui 053000, Hebei, China
}

Correspondence should be addressed to Shenglu Huo; huoshenglu83@126.com

Received 10 March 2021; Revised 24 April 2021; Accepted 27 April 2021; Published 20 May 2021

Academic Editor: Mian Ahmad Jan

Copyright (C) 2021 Shisen Li et al. This is an open access article distributed under the Creative Commons Attribution License, which permits unrestricted use, distribution, and reproduction in any medium, provided the original work is properly cited.

In the field of martial arts, athletes can win the initiative in the competition if they can correctly and timely acquire the field knowledge, evaluate the situation efficiently, and formulate a suitable strategy. In this paper, we use fuzzy mathematics, mathematical statistics, and artificial intelligence learning algorithms to carry out systematic and in-depth research on the selection of Wushu competition scene decision-making. The fuzzy mathematics theory is combined with the intelligent design theory for decision-making based on a multiagent, case-based reasoning selection, and adaptability evaluation analysis. The Wushu competition scene decision system is constructed based on artificial intelligence learning algorithms. Our approach outperforms the existing approaches in terms of accuracy, sensitivity, specificity, and Matthew's correlation coefficient (MCC). The results of our proposed model can be anticipated to have the potential for better flexibility and scalability in martial arts competition.

\section{Introduction}

Wushu is a unique traditional project in China and a treasure in the Chinese nation's cultural heritage. As a sport, the Wushu routine is composed of different styles of technical movements. It has the connotation of attack and defense philosophy and high ornamental value, which gives people reasonable enjoyment [1]. Besides, the Wushu routine is highly praised for its rich and diverse cultural forms. Combining competitive Wushu routine and western competitive sports culture makes daily activities more effective. With the increasing influence of Wushu routines globally, more and more international players begin to practice Wushu routines and participate in international competitions. The combination of Wushu routines and the Olympic concept of higher, faster, and stronger makes the routine competition more accumulated. Therefore, it is necessary to improve the technical level of athletes through scientific training.

In the past, the research on the development of the Wushu routine mainly focused on the situation analysis, qualitative analysis, developing direction discussion, and future developmental trend prediction, among others. However, there is little research on the influencing factors of competitive Wushu routine competition performance, and there is almost no research on the factors influencing athlete's competition performance from the perspective of exercise level $[2,3]$. Therefore, the athlete's movement requires measures that improve the classification of movement patterns into significant groups and support specialists in their decisions at the training.

In this context, the increasing importance of artificial intelligence learning algorithms has led to a substantial contribution. Artificial intelligence learning can simulate and extend human intelligence. These algorithms allow the development of theory, methods, and application systems for simulating and expanding human intelligence. One of its main goals is to enable machines to perform complex tasks that usually require human intelligence. Its rapid development is widely used in military, financial, management, and other fields. The objective of this paper is to build an artificial intelligence assistant system that is suitable for martial arts 
competition decision-making through expert system platform, data mining, online analysis, and virtual reality [4-6]. The artificial intelligence decision support system (IDSS) is a computer intelligent system with special knowledge and inherited experience. It combines artificial intelligence methods and a decision support system (DSS) [7-11]. Numerous researchers have carried out a lot of research on IDSS. In [12], the authors put forward the Web Intelligent Decision Support System framework, which provides effective decision support for decision-makers. In [13], the authors constructed data management, decision-making, and modeling based on a rough set theory that combines an artificial neural network with an expert system to generate new rule's knowledge and realize a fuzzy decision support system. In [14], the authors integrated the agent into an intelligent decision support system and proposed a new coordination engine and information interaction algorithm based on multiagent. Based on multiagent tasking, intelligent decision support system architecture was proposed that improved the intelligent decision-making system's reliability and effectiveness [15]. In [16], an intelligent decision support system for the drilling process based on case-based reasoning was proposed to solve information inaccuracy [16].

However, there is little research on the influencing factors of competitive Wushu routine competition performance, and there is almost no research on the factors influencing athlete's competition performance from the perspective of exercise level $[2,3]$. The major contributions of this paper are as follows:

(1) We comprehensively use fuzzy mathematics, mathematical statistics, and artificial intelligence learning algorithms to carry out systematic and indepth research on the selection of Wushu competition's scene decision-making.

(2) The fuzzy mathematics theory is combined with the intelligent design theory for decision-making based on a multiagent, case-based reasoning selection, and adaptability evaluation analysis. Finally, the Wushu competition scene decision system is constructed based on artificial intelligence learning algorithms.

The rest of this paper is organized as follows. Section 2 discusses the preliminary concept of the system by focusing on different methods followed by artificial intelligence decision-making method for adaptability evaluation of Wushu competition in Section 3. Section 4 presents performance measurement metrics. Results and discussions are presented in Section 5. Finally, Section 6 concludes the paper with a summary and future research direction.

\section{The Preliminary Conception of the System}

The preliminary conception system of the Wushu competition scene decision is divided into three levels, i.e., database layer, processing layer, and display layer. The database layer is the basic support platform, which provides data support for the processing layer. The processing layer is the core executive organization. It is responsible for the logic processing between martial arts tactics. The display layer aims to transform the processed data into decision-making [17]. The library at this layer mainly completes the collection of Wushu tactical data and constructs the Wushu tactical data warehouse. Data warehouse refers to the effective integration of multiple heterogeneous data sources. After integration, it is reorganized according to the theme. It is mainly used to support decision-making and analysis-oriented data processing. Through the collection of martial arts tactical data, the artificial intelligence assistant system completes the collection and storage of basic data, training data, field data, and image document data, including the feature database of Wushu athletes, the feature database of coaches, and the case database of tactics. The martial arts tactics analysis chart is shown in Figure 1.

It can be observed from the figure that the data of martial arts tactics analysis are collected from different angles and levels through statistical reports and tactical deduction. These include the individual data of athletes, coaches, and sports researchers and all kinds of conventional statistical data and field statistics. The decision analysis in the display layer is to establish various topics, analyze the data of different topics, obtain the optimal scheme, and assist the coach in decision-making. The tactical deduction at the performance layer refers to the simulation and display of position and attack and defense routes at your side and the other side in the competition field by electronic means. It reflects the tactics adopted by your side and the other side and analyzes your side's best attack route and defensive line.

\section{Artificial Intelligence Decision-Making for Adaptability Evaluation of Wushu Competition}

The adaptability evaluation of complex Wushu competition involves numerous factors, which are difficult to choose. Due to the complexity of Wushu movements, the competitions are often faced with multiple uncertain factors. The artificial intelligence method can analyze the influence of complex factors and can be used to evaluate the adaptability of the Wushu competition effectively. Based on the theory and practice of fuzzy multiagent and case-based reasoning, the artificial intelligence decision-making of Wushu competition scene adaptability evaluation is proposed here.

3.1. Evaluation Method of Wushu Competition Adaptability Based on Fuzzy Mathematics. Fuzzy theory can simulate human thinking, and it is feasible for human decisionmaking. The characteristics of a fuzzy membership relationship in fuzzy theory can be applied to many decisionmaking fields. At present, fuzzy subordinate decisionmaking has been successfully obtained in considerable scientific research. The field adaptability evaluation of Wushu competition under complex conditions is affected by various quantitative and qualitative uncertain factors, such as Wushu action design, routine design, field performance status, and equipment problems. Due to these factors' different units and attributes, it is not easy to compare them. Besides, it is one of the complex problems in adaptability 


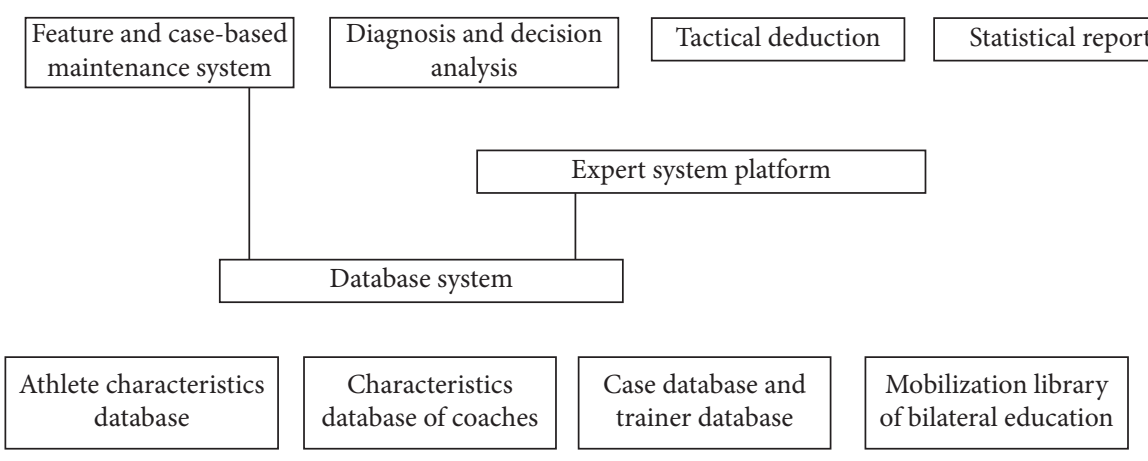

FIgURE 1: A system for analyzing martial arts tactics.

evaluation to select the main or critical influencing factors from so many factors and then use some measurable indicators to describe them. For the problem of commensurability of the evaluation index, the fuzzy mathematics method can be effectively solved. Because of these factors affecting the adaptability evaluation of the Wushu competition scene, the results obtained by using the fuzzy comprehensive evaluation method are more accurate and applicable [18].

3.2. The Fuzzy Comprehensive Evaluation Model. Assuming that the evaluation target set $O=\left\{O_{1}, O_{2}, \ldots, O_{m}\right\}$ and the evaluation index set factory is equal to network $U=\left\{U_{1}, U_{2}, \ldots, U_{m}\right\}$, then the basic model of a fuzzy comprehensive evaluation is as follows:

$$
D=\left\{w_{1}, w_{2}, \ldots, w_{m}\right\} \times\left[\begin{array}{cccc}
r_{11} & r_{12} & \ldots & r_{1 n} \\
r_{21} & r_{22} & \ldots & r_{2 n} \\
\vdots & \vdots & \ddots & \vdots \\
r_{m 1} & r_{m 2} & \ldots & r_{m n}
\end{array}\right],
$$

where $D$ is the set of comprehensive evaluation values and $O_{i}$ is the fitness of evaluation objectives. The weight of the evaluation index is determined by the analytic hierarchy process (AHP). AHP is a multiobjective decision analysis method combining quantitative and qualitative analysis. It combines mathematical processing with human experience and subjective judgment and can effectively analyze the nonsequential relationship between the levels of objective criteria system, the judgment, and decision-making of comprehensive measurement evaluation. The method to determine the weight of the evaluation index is as follows [19].

According to the evaluation, objectives are achieved based on the mutual influence and subordination relationship among evaluation indexes. The hierarchy of indicators is divided into the target layer, criterion layer, and index layer from high to low (Figure 2). The indicators of the upper level play a dominant role in the indicators of the next level.

For the difference of the influence of pairs of indicators, the judgment matrix constructed according to the above method can objectively reflect. Still, when analyzing and comparing all the results of the indicators, there will inevitably be some degree of inconsistency. To eliminate the inconsistency, the elements in the judgment matrix A should satisfy the following conditions:

$$
a_{i j} a_{j k}=a_{i k}, \quad \forall i, j, k=1,2,3, \ldots, n .
$$

3.3. Fuzzy Membership Function of Evaluation Index. The relationship between fuzzy set and element set can be expressed by any interval value, i.e., 0,1 . When the fuzzy set completely contains the element, it is expressed as " 1 "; when the fuzzy set does not contain the element, it is expressed as "0". Similarly, the membership degree indexes the uncertainty of the membership relationship between element sets and fuzzy sets. Generally, when solving a practical problem, the first thing is to determine the membership function, which is usually approximately determined by the reasoning method. The connotation of the subordinate function is to reflect the objective law into the function formula and to synthesize, process, reorganize, and transform people's subjective consciousness. Therefore, the determination of membership function should conform to objective law and have a certain degree of subjectivity and experience, not through subjective imagination. The membership function can be determined by the intuitionistic method, fuzzy statistical method, preferred comparison method, binary comparison method, and absolute comparison method.

3.4. The Intelligent Decision Support System. An intelligent decision support system (DSS) makes use of the advantages of machine learning and expert system technology in qualitative analysis and uncertain reasoning, makes full use of human experience and knowledge in problem-solving, and provides a new way to solve the above problems. The expert system establishes a domain expert knowledge base and problem-solving base system. The expert system can make the machine intelligence reach or even surpass the human expert level in some aspects. The application of unified technology in management often aims at the decision-making problems in specific fields. Applying expert system technology to decision support system and establishing an intelligent decision support system (DSS) can overcome the limitations of expert system and decision support system. Better support management decision service 


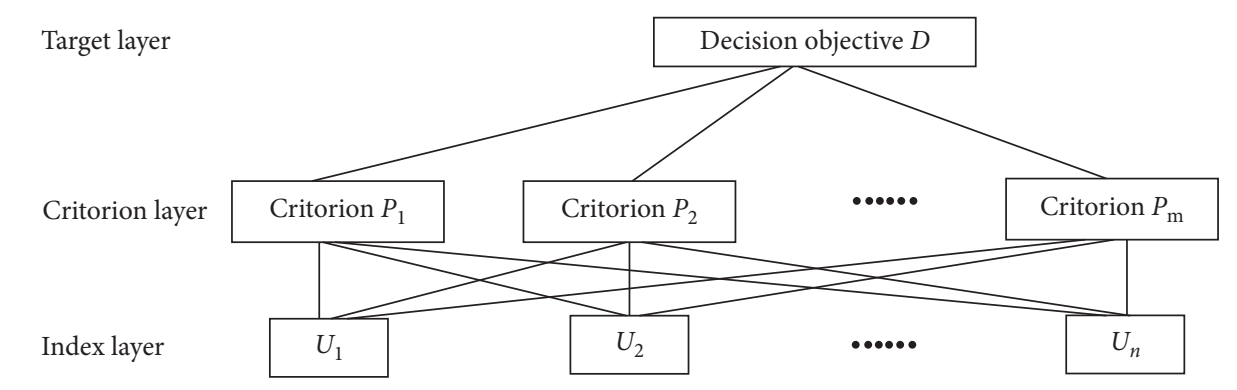

FIgURE 2: Hierarchical model.

artificial intelligence and expert system technology not only have a profound impact on the technology and structure of decision support system but also have a profound impact on the concept of decision support system in recent years. Almost all the research studies on DSS are based on the application of machine learning technology, and the combination of expert system and decision support system is a direct body. Now, the intelligence of all parts of DSS, such as the combination of man-machine dialogue components, the combination of solving methods, the combination of database model base, method base, and their management system. The combination of expert system technology and decision support component improves the function of each component, which will undoubtedly greatly improve the decision supportability [20-23].

With the deepening of research, expert system technology has penetrated all aspects of DSS, such as architecture and problem-solving, which has an important impact on decisionmaking methods and processes. The research of intelligent decision support system has gradually developed from the expansion of the function of decision-making components in the past to the comprehensive integration of components and from the past quantitative model to the intelligent decisionmaking method based on knowledge, and it makes the theory and method of intelligent decision support system mature gradually. Turbo has studied the implementation of a decision support system $1 \%$, but the intelligent system implementation method cannot guarantee that the developed system is an intelligent decision support system.

The intelligent decision support system (IDSS) is a kind of computer intelligent program system with special knowledge and experience. It is the combination of an expert system (ES) and a Wushu competition scene decision support system (DSS). Combining the characteristics of both qualitative and quantitative analysis can be organically combined, and the ability and scope of solving problems can be improved. As shown in Figure 3, it is the structure diagram of the integration of the expert system and decision support system of the Wushu competition scene.

It can be seen from the figure that the combination of DSS and ES in IDSS is mainly reflected in the following three aspects:

(1) The overall combination of DSS and ES

(2) The combination of $\mathrm{KB}$ and $\mathrm{MB}$

(3) The combination of the static database (DB) and dynamic database

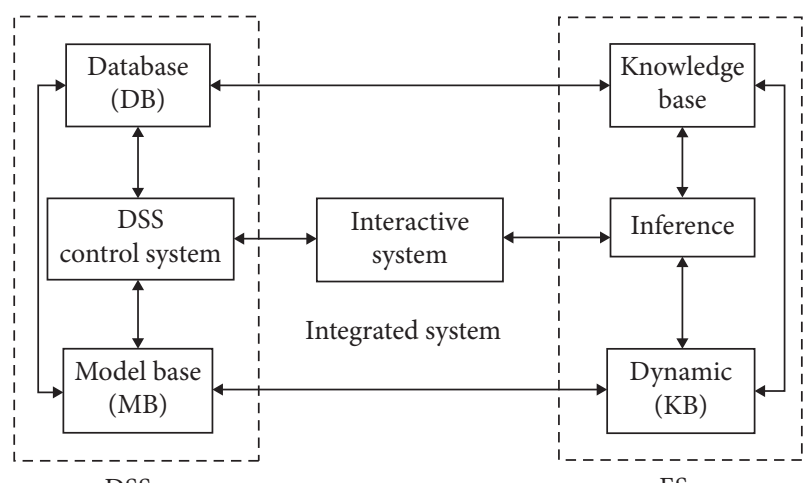

DSS

Figure 3: Structural chart for integrating expert system and decision support system.

3.5. Deep Neural Network Model. A deep neural network model is a multilayer network of processing nodes and is most widely used in industries, simulation games, robotics, and many other different fields. A deep neural network model needs to be built based on the huge amount of data. The deep model can be established based on various relationships and laws analyzed by data mining and can also be established by combining martial arts professional knowledge and the experience of coaches and experts. Valuable information can be obtained from a large number of data through the model, and this information can be displayed in an intuitive form so that the data can play an effective role, and the model can be dynamically increased and improved through practical application. Besides, tactical deduction, virtual arena, virtual exhibition hall, and other performance methods are gradually mature.

The basis of the deep neural network assistant system of Wushu competition decision-making is also a large amount of data. It is precisely because of the large amount of data and information accumulated in the daily training and actual competition, which has laid a solid foundation for the system. Also, the rich knowledge and on-the-spot command experience of the coaches provide reliable support for the adjustment and optimization of the system. The data mining, online analysis, and virtual technology involved in this system are relatively mature technologies and are widely used in military, economic, management, and other fields, and achieved success.

\section{Formulation of Measurement Scales}

To evaluate the performance of a machine learning algorithm, four parameters are commonly used to check the 
model reliability and validity. These parameters include overall accuracy of the model, specificity as true-negative rate, sensitivity as true-positive rate, and MCC as Mathew's correlation coefficient. These four metrics calculation formulas are as follows:

$$
\begin{aligned}
\mathrm{ACC} & =1-\frac{m_{-}^{+}+m_{+}^{-}}{m^{+}+m P^{-}}, \quad 0 \leq \mathrm{ACC} \leq 1, \\
\text { specificity } & =1-\frac{m_{+}^{-}}{m^{-}}, \quad 0 \leq \mathrm{Sp} \leq 1, \\
\text { sensitivity } & =1-\frac{m_{-}^{+}}{m^{+}}, \quad 0 \leq \mathrm{Sn} \leq 1, \\
\mathrm{MCC} & =\frac{1-\left(m_{-}^{+}+m_{-}^{+} / m^{+}+m m^{-}\right)}{\sqrt{\left(1+m_{+}^{-}-m_{-}^{+} / m^{+}\right)\left(1+m_{-}^{+}-m_{+}^{-} / m^{-}\right)}}, \quad-1 \leq \mathrm{MCC} \leq 1,
\end{aligned}
$$

where $m^{+}$represents true positive, $m^{-}$represents the total true negative, $m_{-}^{+}$represents the false positive, and $m_{+}^{-}$ represents the false negative.

In the field of machine learning, the entity that can work independently and has the interactive ability is generally defined as an agent. Independent traditional agents are limited in enjoying resources and solving problems. When they encounter complex and huge practical decision-making problems, they often cannot solve them effectively. Based on this, scholars at home and abroad put forward a multiagent system. The traditional multiagent system is a kind of distributed multiagent system. It can effectively solve complex and huge practical decision-making problems, which are not possessed by independent traditional agents. Through multiple in different hosts or locations, the traditional multiagent can complete more complex decision-making tasks through the interaction and cooperation between the independent traditional multiagents running on.

\section{Experimental Results and Discussion}

In this section, we evaluate and discuss the performance of our proposed model in detail. There are a lot of professional data in martial arts teaching and scientific research, such as daily training data, competition data, teaching data, and experience data of domestic and foreign counterparts. With the help of deep neural network (DNN), these data are effectively classified and sorted out to form a data warehouse, and an artificial intelligence assistant system for decisionmaking on the spot of Wushu competition is constructed.

5.1. Interindividual Classification. The establishment of the model needs to be based on the experience of teaching and training level experts; on the other hand, it is to model through the association relationship obtained by data mining. The model includes a static model and a dynamic model. The static model is mainly composed of the individual ability model of the players and the overall ability model of the players on the field. The dynamic model needs to take the field parameters as the input conditions and then carry out the field operation model as shown in Table 1. For example, the formation influence model of both sides can analyze the attack and defense of the current formation by comparing the positions of the players on the field and the positions of the opposing team members. By inputting the field data of both players in real time, it can analyze which position of the team needs to be strengthened and which position of the other side is the weakest. Using artificial intelligence algorithm [24, 25] for selflearning and self-improvement of the model, on the one hand, can optimize and adjust various models through selflearning ability; on the other hand, according to the feedback data in practical application, the key parameters are modified to make the model more in line with the actual situation.

5.2. Individuality of Movement Patterns Multiple Agents. Multiple agents in the system will produce a variety of different decision-making schemes. Before making decisions on the schemes, they must be ranked first; then, the cooperation and cooperation among multiple agents in the system are needed to obtain a higher reliable solution. At present, a large number of studies on the priority of decision schemes have been carried out, and fruitful results have been obtained. For example, some scholars have applied scientific research on the area of fuzzy numbers to the order of the pros and cons of decision-making schemes; some scholars have combined the Minkowski function with grey number theory and put forward the corresponding ranking method. Some scholars use the local rough set to sort the decision schemes in the stochastic control model. However, all of the above methods have their limitations. On the problem of ranking the advantages and disadvantages of the decisionmaking schemes, the system has not been fundamentally and effectively solved in the application process, and there are still many problems in the ranking of schemes, which makes the evaluation results to lack of scientific rationality, as shown in Table 2. Figures 4-7 show the comparative experimental results of different methods on accuracy (ACC), sensitivity (Sn), specificity (Sp), and Matthew's correlation coefficient (MCC).

At the same time, different from the independent traditional agent, the traditional multiagent system can complete the adaptive adjustment according to the change of environment, which makes it with a strong adaptability feature. The traditional multiagent system is a network system composed of several loosely coupled agents. The traditional multiagent system provides a new framework and model for solving complex problems. It has the performance that other systems cannot match. It has the advantages of distribution, learning and reasoning ability, independence, cooperation, and self-organization ability. 
TABle 1: Performance of the individual ability model of the players.

\begin{tabular}{|c|c|c|c|c|}
\hline Classification task & $\operatorname{ACC}(\%)$ & Sn $(\%)$ & Sp (\%) & $\overline{\mathrm{MCC}}$ \\
\hline Strikes ground & 92.81 & 91.72 & 94.12 & 0.821 \\
\hline Strikes body & 93.23 & 92.4 & 96.3 & 0.879 \\
\hline Distance time & 94.65 & 93.3 & 95.52 & 0.831 \\
\hline Strikes kicks attempts & 92.07 & 91.1 & 93.7 & 0.812 \\
\hline Strikes attempts & 93.49 & 90.6 & 95.91 & 0.799 \\
\hline
\end{tabular}

TABLE 2: Performance of the individual movement patterns multiple agents.

\begin{tabular}{lcccc}
\hline Classification task & ACC (\%) & Sn (\%) & Sp (\%) & MCC \\
\hline Kicks attempts & 92.81 & 91.72 & 93.12 & 0.851 \\
Punches attempts & 93.23 & 92.4 & 93.88 & 0.838 \\
Side control time & 92.65 & 91.08 & 93.64 & 0.825 \\
Leg kicks & 94.07 & 93.76 & 94.4 & 0.812 \\
Head kicks & 94.49 & 94.44 & 95.16 & 0.891 \\
\hline
\end{tabular}

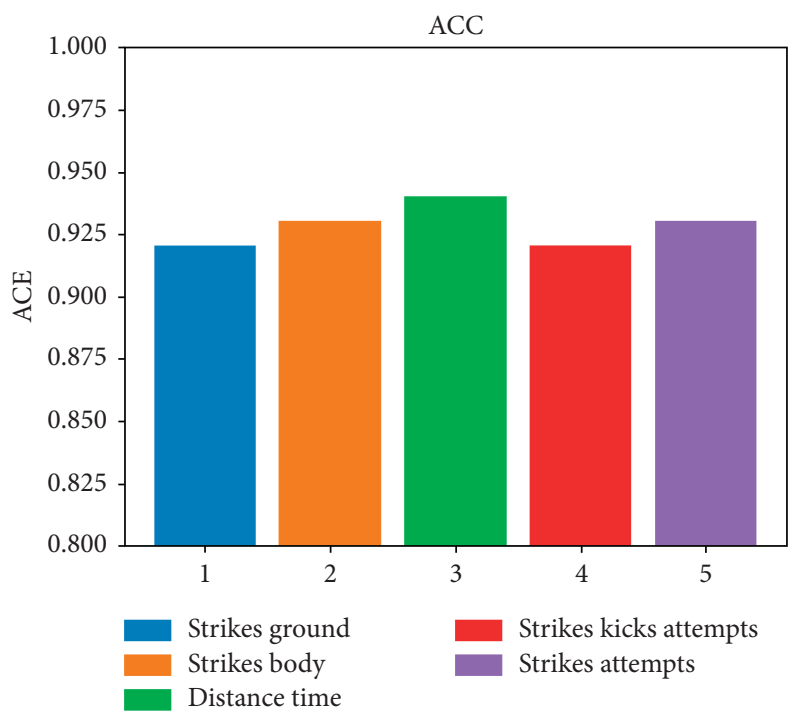

Figure 4: Comparative experimental results of different methods on accuracy.

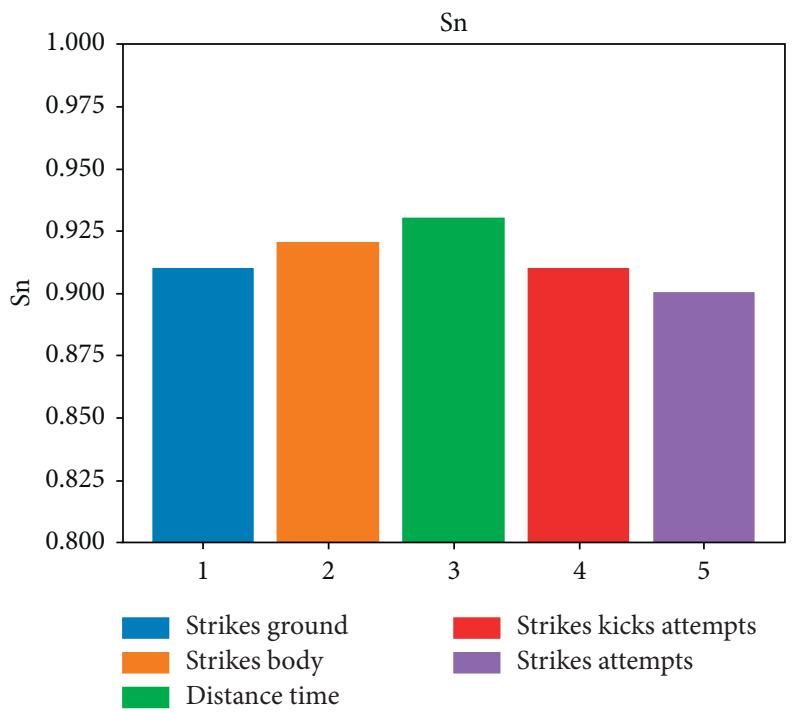

FIGURE 5: Comparative experimental results of different methods on sensitivity. 


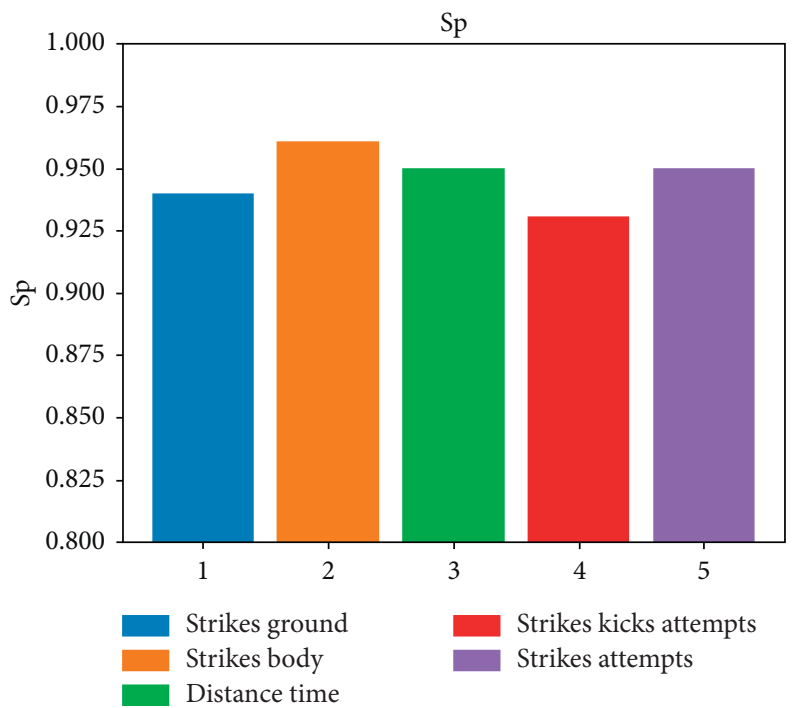

FIGURE 6: Comparative experimental results of different methods on specificity.

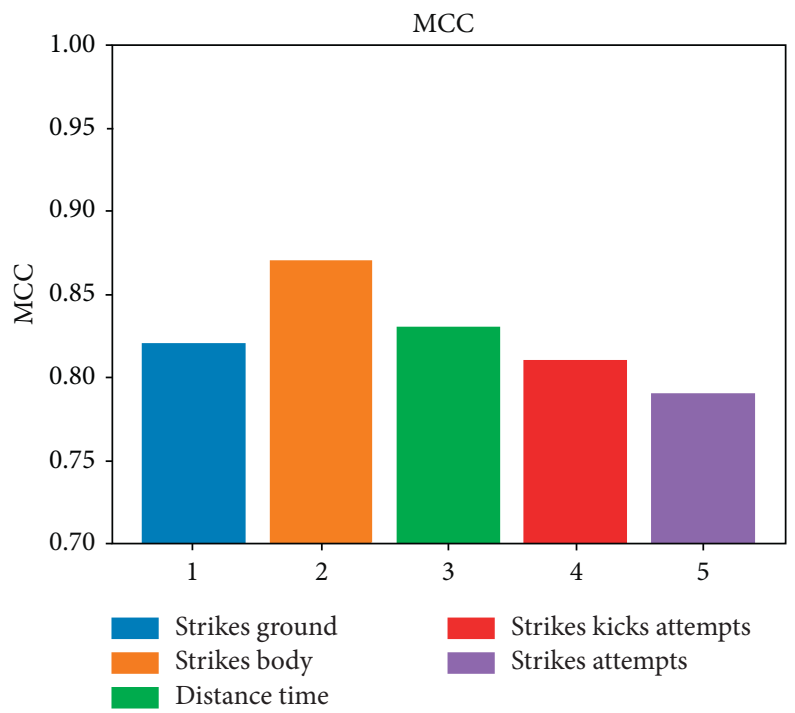

FIgURE 7: Comparative experimental results of different methods on MCC.

\section{Conclusion}

With abundant data and multiple analysis tools, the machine learning-assisted system of Wushu competition can display the real-time data of Wushu competition. It can assist the coaches in analyzing and making decisions on the spot and put forward targeted training objectives for daily training, which has substantial application value. On this basis, it can also build and establish numerous application systems. In this research, we have proposed a special system for martial arts experts to collect and accumulate the experience and knowledge of experts and scholars in martial arts to improve the competitive level, teaching level, and scientific research level of Chinese martial arts. Moreover, large-scale distributed control is adopted in a multiagent system which does not affect the operation of the whole multiagent. Our model has much better accuracy, sensitivity, specificity, and Matthew's correlation coefficient (MCC) in comparison with the existing schemes which shows its applications in Wushu competition. The proposed model results can be anticipated to have the potential for better flexibility and scalability in martial arts competition.

\section{Data Availability}

The data used to support the findings of this study are included within the article.

\section{Conflicts of Interest}

The authors declare that they have no conflicts of interest.

\section{References}

[1] K. Wang, "An empirical analysis of the popularization development of Taijiquan based on the concept of national 
fitness," Journal of Wuxi Vocational and Technical College, vol. 16, no. 1, pp. 70-73, 2017.

[2] C. Wang, Research on the Development of Bengbu Martial Arts under the Background of National Fitness, Huaibei Normal University, Huaibei, China, 2018.

[3] L. Zhang, Research on the Current Situation and Countermeasures of Taijiquan Teaching at Jilin University, Jilin University, Changchun, China, 2014.

[4] J. Shi and Y. Xue, "Research on the development of Taijiquan in Chengdu I-taking the 13th Taijiquan championship in Chengdu in 2016 as an example," Sichuan Sports Science, vol. 36, no. 2, 2017.

[5] X. Chai, C. Gao, and Y. Jing, The Promotion and Development Status of Taiji Aerobics under the National Fitness Program [Chuan], Vol. 10, China Good University, Beijing, China, 2017.

[6] Z. Shi, Advanced Artificial Intelligence, Science Press, Beijing, China, 2011.

[7] R. He, L. Chen, B. Wan et al., "Research on DSS oriented model base system based on agent," Computer and Digital Engineering, vol. 39, no. 12, pp. 54-57, 2011.

[8] J. Zhu and Y. Liu, "The construction method of an intelligent decision support system based on multi-agent," Journal of Beijing University of Technology, no. 1, pp. 6-10, 2001.

[9] S. Zhang, B. Li, and X. Zhao, "Application of intelligent decision support system in ventilation system optimization of Zhaozhuang mine," Modern Mining, vol. 34, no. 8, pp. 118$122,2018$.

[10] X. Li, "Analysis of Taijiquan fitness in China from the perspective of national fitness," Foreign Teachers of China, no. 6, pp. 46-47, 2018.

[11] G. Xu, "Exploration and research on the cultivation of ways of teenagers' Wushu enlightenment training interest," Contemporary Sports Science and Technology, vol. 7, no. 2, pp. 43-44, 2017.

[12] Y. Xu, X. Han, and L. Yang, "Research on the development path of traditional Wushu into the campus," Chinese Wushu. Research, no. 7, pp. 61-63, 2018.

[13] Y. Qiu, "On the inheritance of traditional martial arts to confucian spiritual cultivation methods," Sports Science Research, vol. 18, no. 5, pp. 15-18, 2014.

[14] B. Liu, "The development of martial arts under the guidance of new era thoughts. Chinese martial arts," Research, vol. 7, no. 8, pp. $45-48,2018$.

[15] Y. Zhang and J. Huang, "The influence of martial arts novels and film and TV works on young people's learning martial arts," Sports Research and Education, vol. 30, no. 2, pp. 98100, 2015.

[16] X. Su, P. Li, and W. Shi, "On the characteristics of Chinese Wushu development during the cultural revolution," Journal of Wuhan Institute of Physical Education, vol. 33, no. 6, pp. 29-32, 1993.

[17] S. Xiong, Research on Fuzzy Portfolio Optimization and Application, Harbin Institute of Technology, Harbin, China, 2014.

[18] A. Dai, Children's Sensitive Period Diagnosis System Based on Axiomatic Fuzzy Decision Tree, Jiangxi University of Finance and Economics, Nanchang, China, 2017.

[19] T. Satty, The Analytic Hierarchy Process, McGraw-Hill, New York, NY, USA, 1980.

[20] X. Wang, Y. Hong, J. Huang, and Z. P. Jiang, “A distributed control approach to a robust output regulation problem for multi-agent linear systems," IEEE Transactions on Automatic Control, vol. 55, no. 12, pp. 2891-2895, 2010.
[21] X. Wang, M. Yang, Y. Jiang et al., "On self-tuning networkson-chip for dynamic network-flow dominance adaptation," ACM Transactions on Embedded Computing Systems (TECS), vol. 13, no. 2, pp. 1-21, 2014.

[22] J. Li, Research on Some Problems of Multi-Agent System Unification, School of automation, Nanjing University of Technology, Nanjing, China, 2011.

[23] W. Liu, Coordinated Control and Optimization of Complex Multi-Agent Networks, School of automation, Huazhong University of science and technology, Wuhan, China, 2011.

[24] W. Cai, B. Liu, Z. Wei, M. Li, and J. Kan, "TARDB-net: tripleattention guided residual dense and BiLSTM networks for hyperspectral image classification," Multimedia Tools and Applications, vol. 80, no. 7, pp. 11291-11312, 2021.

[25] X. Zhang, Y. Yang, Z. Li, X. Ning, Y. Qin, and W. Cai, "An improved encoder-decoder network based on strip pool method applied to segmentation of farmland vacancy field," Entropy, vol. 23, no. 4, p. 435, 2021. 